\title{
Probing the Electronic Properties of Trimesic Acid Nanoporous Networks on Au(111)
}

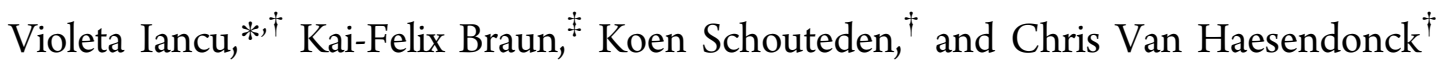 \\ ${ }^{\dagger}$ Laboratory of Solid-State Physics and Magnetism, KU Leuven, BE-3001 Leuven, Belgium \\ ${ }^{\ddagger}$ Iot-labs, 64293 Darmstadt, Germany
}

ABSTRACT: Nowadays molecular nanoporous networks have numerous uses in surface nanopatterning applications and in studies of host-guest interactions. Trimesic acid (TMA), a benzene derivative with three carboxylic groups, is a marvelous building block for forming $2 \mathrm{D} \mathrm{H}$-bonded porous networks. Here, we report a low-temperature study of the nanoporous "chicken-wire" superstructure formed by TMA molecules adsorbed on a $\mathrm{Au}(111)$ surface. Distinct preferential

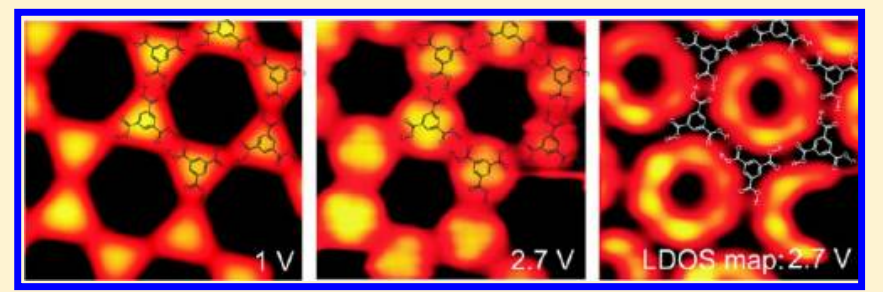
orientations of the porous networks on $\mathrm{Au}(111)$ lead to the formation of peculiar TMA polymorphs that are stabilized only at the boundary between rotational molecular domains. Scanning tunneling microscopy (STM) and spectroscopy are used to investigate the electronic properties of both the molecular building blocks and the pores. Sub-molecular-resolution imaging and spatially resolved electronic spectroscopy reveal a remarkable change in the appearance of the molecules in the STM images at energies in the range of the lowest unoccupied molecular orbital, accompanied by highly extended molecular wave functions into the pores. The electronic structure of the pores reflects a weak confinement of surface electrons by the TMA network. Our experimental observations are corroborated by density-functional-theory-based calculations of the nanoporous structure adsorbed on $\mathrm{Au}(111)$.

\section{INTRODUCTION}

Molecular architectures on surfaces have attracted much attention lately because of their multifunctional character. In particular, porous molecular networks have recently become increasingly appealing because of their potential in surface patterning ${ }^{1-6}$ and electronic-state engineering ${ }^{7,8}$ at both solidvacuum and solid-liquid interfaces. Their functionality can be tailored even further by tuning the reactivity of the underlying surfaces as well as by changes in their immediate environment. Trimesic acid (TMA) molecules, which are benzene derivatives with three carboxylic groups, are often used as metal-organic framework linkers for high-surface-area applications. ${ }^{9,10}$ They are excellent building blocks for forming 2D H-bonded porous networks on low-reactivity surfaces. ${ }^{4,11,12}$ Previous studies successfully tested the TMA networks for their ability to host small molecules in the pores, ${ }^{2,4,5,11}$ relying on scanning tunneling microscopy (STM) imaging at solid-liquid or solid-vacuum interfaces. However, little is known about the electronic properties of the TMA molecules, and even less is known about the nanometer-sized voids that expose the underlying surface. For example, two different appearances of TMA in the STM experiments have been reported so far, but a physical explanation is still lacking. ${ }^{4,11-13}$ STM is both a topographic and an electronic tool; therefore, detailed knowledge of the electronic properties of materials under investigation is of crucial importance for a correct interpretation of the STM images. A profound understanding of the structural and electronic characteristics of the pores/molecules is of the utmost importance in designing future single or multicomponent host/guest systems.

Here, we unravel the electronic properties of TMA molecules and their interaction with the underlying $\mathrm{Au}(111)$ surface. We probe the low-molecular-coverage regime where the molecules form cyclic dimers by double $\mathrm{H}$-bonds between their carboxylic groups, yielding the so-called "chicken-wire" structure. ${ }^{11,12}$ The observed structure is a result of the balanced interplay between molecule-molecule and molecule-substrate interactions. The periodic modulation of the energy landscape results in distinct preferential orientations of the network structure relative to the substrate. Peculiar TMA polymorphs such as pentamers and heptamers are stabilized at the boundary between rotational molecular domains. Using STM and scanning tunneling spectroscopy (STS) at low temperature, we detect the lowest unoccupied molecular orbital (LUMO) and identify a remarkable change in the appearance of the TMA molecules that can be related to LUMO-mediated electron tunneling. The electronic structure of the pores is dominated at LUMO energies by the spatially extended molecular orbitals and displays a weak confinement of surface electrons by the TMA network. Density functional theory (DFT)-based calculations that include van der Waals forces are employed to model the nanoporous TMA structure formed on the surface and to calculate its properties and the interfacial charge transfer. The

Received: February 25, 2013

Revised: August 9, 2013

Published: August 14, 2013 
simulated STM images are in excellent agreement with the experiment and capture the change in the experimentally detected appearance of the molecule.

\section{EXPERIMENTAL SECTION}

The growth of the TMA adlayers was performed under ultrahigh vacuum (UHV) conditions. TMA powder was purchased from SigmaAldrich and was sublimated at $445 \mathrm{~K}$ using a Knudsen cell. Submonolayer amounts of TMA molecules were dosed onto a clean $\mathrm{Au}(111) /$ mica substrate held at room temperature and subsequently annealed at $380 \mathrm{~K}$ for $15 \mathrm{~min}$. The molecular structures were characterized using a low-temperature STM system (Omicron) operated at $4.5 \mathrm{~K}$. Scanning tunneling spectroscopy (STS) was performed by lock-in detection using amplitudes of 4 to $40 \mathrm{mV}$ at 746 Hz. Distance-voltage $\mathrm{d} z / \mathrm{d} V(V)$ spectroscopy was employed for large bias voltages and measured with a closed feedback loop. ${ }^{14}$ For STM tips, we used polycrystalline $\mathrm{W}$ wires. The tips were electrochemically etched and cleaned in situ by thermal treatment. All bias voltages mentioned are with respect to the sample, and the STM tip is virtually grounded. The STM images were analyzed using the WSxM software $^{15}$ and the STWS program. ${ }^{16}$

\section{COMPUTATIONAL DETAILS}

To explain and complement the experimental observations, we performed DFT-based calculations implemented in the Quantum ESPRESSO code (version 4.3.2). ${ }^{17}$ We modeled the TMA nanoporous network using a hexagonal unit cell comprising an $\mathrm{R}_{2}^{2}(8)$ dimer. To mimic the $\mathrm{Au}(111)$ surface, we used a four-layer slab with the in-plane unit cell consisting of 36 gold atoms placed in a $6 \times 6$ configuration $(17.3 \AA \times 17.3 \AA)$. To be in agreement with the experimental results, the TMA structure was constructed such that the TMA dimer is oriented parallel to the [211] direction of the substrate. The periodicity of the calculated structure is imposed by the slab unit cell to be $1.73 \mathrm{~nm}$, which implies $\mathrm{OH} \cdots \mathrm{O}$ bonds of $2.8 \AA$. Single noninteracting TMA molecules were modeled using a $5 \times 5$ gold slab. For geometrical optimizations, we employed the $\mathrm{vdW}-\mathrm{DF}^{18}$ functional with a revPBE exchange that accounts for nonlocal van der Waals forces. $^{19}$ The Perdew-Burke-Ernzerhof $(\mathrm{PBE})^{20}$ functional is also used for comparison and when mentioned to calculate the system properties while fixing the coordinates to the vdW-DF-optimized coordinates. Geometrical relaxations were carried out until the forces on atoms were less than $0.02 \mathrm{eV} / \AA$ while the gold atoms were fixed. The calculations were performed using an energy cutoff of $490 \mathrm{eV}$ for the wave functions and ultrasoft pseudopotentials. A $k$-point grid of 2 $\times 2 \times 1$ was used for geometrical optimizations and STM simulations. The STM images were computed using the Tersoff-Hamann formalism $^{21}$ for wave functions calculated with the PBE functional. The DFT-based calculation results were visualized using XCrySDen software. ${ }^{22}$

\section{RESULTS AND DISCUSSION}

Self-Assembly of Trimesic Acid Molecules on Au(111): The Role of the Substrate. Figure 1a illustrates an STM image of a nanometer-sized island formed by TMA molecules when adsorbed on $\mathrm{Au}(111)$ surface at low coverage. The $\mathrm{Au}(111)$ herringbone reconstruction is observed in the background and is not affected by the adsorbate layer. The molecules form a hexagonal honeycomb network with two molecules per unit cell (Figure $1 \mathrm{~b}$ ). The lattice bears a small distortion of 2 to $4 \%$, and the unit cell vectors average to $1.62 \pm$ $0.05 \mathrm{~nm}$. This porous arrangement of TMA/Au(111), called the "chicken wire", represents the lowest-density structure among many polymorphs that TMA molecules can form on a $\mathrm{Au}(111)$ surface as a function of surface coverage, as previously reported by Ye et al. ${ }^{12}$ Chicken-wire structure formation is dictated by dimeric $\mathrm{R}_{2}^{2}(8) \mathrm{H}$ bonds formed between the carboxylic $(\mathrm{COOH})$ groups of the TMA molecules (Figure 1c),

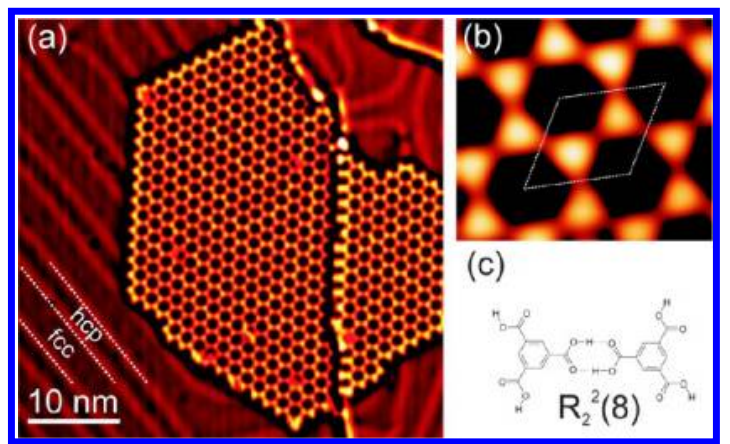

Figure 1. (a) STM image of a TMA island nucleated at a step. Dotted lines mark the discommensuration lines that separate the fcc and hcp areas. (b) Close-up-view STM image with the primitive unit cell drawn. Tunneling parameters: $1 \mathrm{~V}$ and $0.1 \mathrm{nA}$. (c) Chemical structure of the TMA $\mathrm{R}_{2}^{2}(8)$ dimer.

similar to the TMA arrangement in the bulk. ${ }^{9}$ Potential application of the TMA nanoporous structures as 2D templates will benefit from a detailed understanding of their electronic properties. In the following text, we discuss the interaction between the TMA chicken-wire structure and the underlying $\mathrm{Au}(111)$ surface and report on its electronic properties.

First we will discuss the role of the substrate in molecular self-assembly. Determining the registry of the molecules with the substrate is not a straightforward task because atomic resolution and molecular resolution usually exclude each other. Nevertheless, the $\mathrm{Au}(111)$ substrate is known to reconstruct into the $(22 \times \sqrt{ } 3) R 30$ reconstruction and form alternating fcc and hcp regions separated by discommensuration lines or stacking faults (Figure 1a). ${ }^{23}$ These stacking faults are oriented along the $[\overline{2} 11]$ directions of the substrate ${ }^{24}$ and hence allow us to infer the orientation of the molecular islands with respect to the surface $[\overline{2} 11]$ directions. Two preferential orientations of the islands are detected with respect to the [211] surface directions (Figure 2a). Of a total of 60 islands analyzed, 57\% are oriented $4 \pm 1^{\circ}$ and $28 \%$ are oriented $30 \pm 1^{\circ}$ from the $[\overline{2} 11]$ directions. Whenever molecules nucleate at kink steps or at surface dislocations, islands with other orientations are also created, but in smaller numbers (15\%). Step barriers do not seem to affect the molecular organization, and molecules are found on both ascending and descending terraces (Figure 1a).

Figure 2a displays an STM image of a TMA island formed by two domains having the orientations discussed above. A closeup view of the area highlighted by the black rectangle is presented in Figure 2b. Here, one notices a "light" domain wall (DW1) present between the two domains ${ }^{25}$ that consists of TMA pores formed by pentamers and heptamers. These pores are peculiar because the 3-fold symmetry of the TMA molecules does not allow the natural formation of cyclic pentamer and heptamer H-bonded structures. A different type of domain wall is formed between domains of the same orientation. Figure $2 \mathrm{c}$ illustrates a "heavy" domain wall (DW2) created at the junction between two $4^{\circ}$-oriented superstructures. In this case, the two lattices are shifted only by half the intermolecular dimer distance $(0.5 \mathrm{~nm})$. A schematic drawing of the two types of domain walls is presented in Figure $2 \mathrm{~d}$. Both domain walls are thin, which is expected when the molecule-substrate potential is comparable to the moleculemolecule interaction. ${ }^{25}$ The observed preferential orientations of the TMA domains and the formation of domain walls are indicative of a balanced interplay between molecule-substrate 

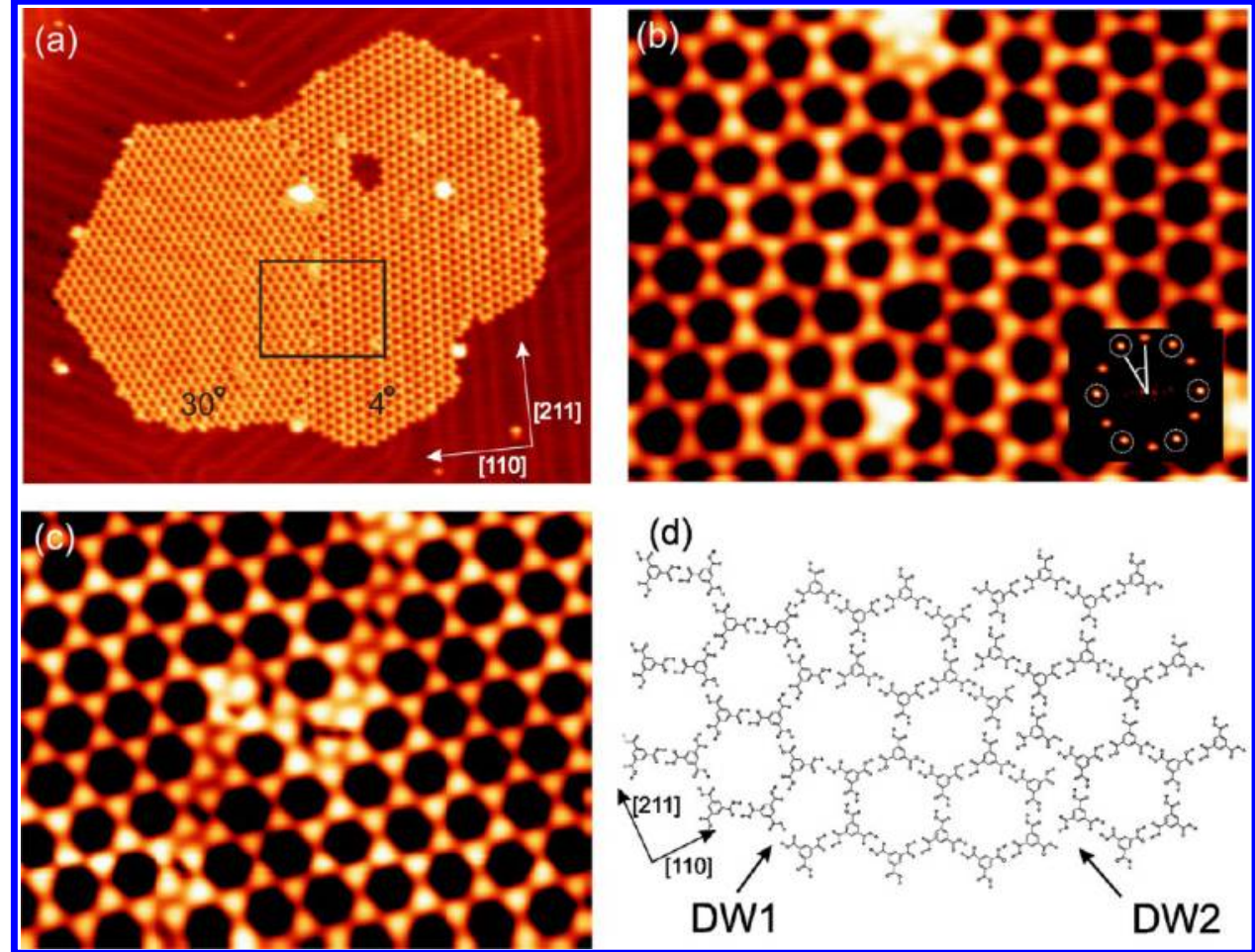

Figure 2. (a) STM image $(85 \mathrm{~nm} \times 70 \mathrm{~nm})$ of a TMA island consisting of two rotational domains. Tunneling parameters: $1.2 \mathrm{~V}$ and $0.19 \mathrm{nA}$. (b) STM image $(19 \mathrm{~nm} \times 14 \mathrm{~nm})$ of the area highlighted in image a, presenting a close-up view of the "light" domain wall (DW1). The inset indicates the fast Fourier transformation that is used to measure the relative angle between the two domains, $26 \pm 1^{\circ}$. (c) STM image $(16 \mathrm{~nm} \times 12 \mathrm{~nm})$ of two TMA domains forming a "heavy" domain wall (DW2). Tunneling parameters: $-0.8 \mathrm{~V}$ and $0.28 \mathrm{nA}$. (d) Schematic drawing of the two types of domain walls.
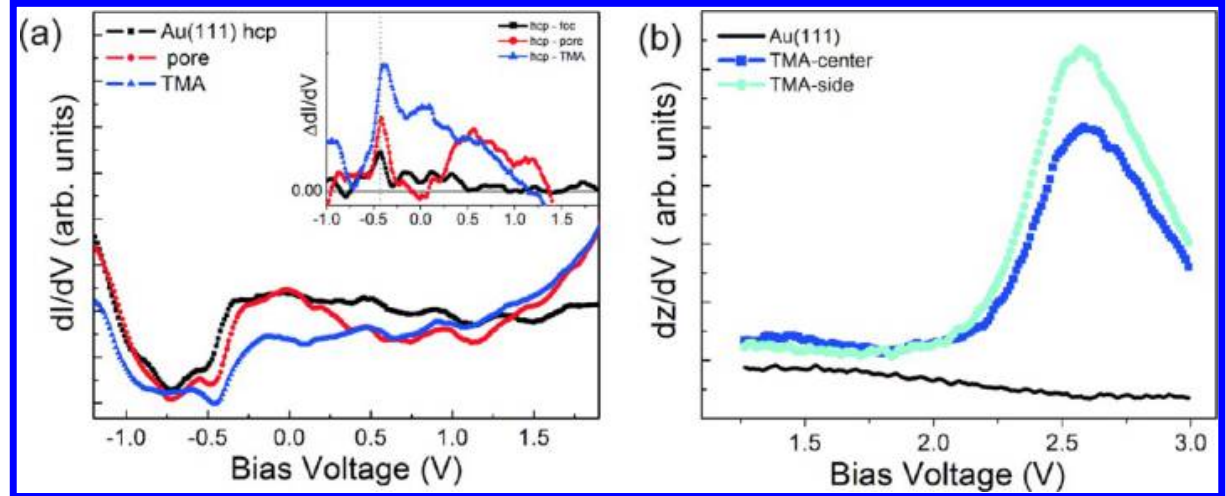

Figure 3. (a) $\mathrm{d} I / \mathrm{d} V(V)$ spectra of bare $\mathrm{Au}(111)$ (in the hcp region), the TMA molecule, and the gold surface inside the pore. The inset illustrates the $\mathrm{d} I / \mathrm{d} V$ difference between the hcp site of the bare $\mathrm{Au}(111)$ and other spectra of interest. The set point is $-1.2 \mathrm{~V}$ and $0.18 \mathrm{nA}$. (b) $\mathrm{d} z / \mathrm{d} V(V)$ spectra on the substrate and on the molecule. The set point is $1 \mathrm{~V}$ and $0.17 \mathrm{nA}$.

and molecule-molecule interactions in the TMA self-assembly on $\mathrm{Au}(111)$.

The role of the substrate is better defined in terms of the commensuration between the solid-phase dimer lengths and the surface. In the solid phase, the $\mathrm{OH} \cdots \mathrm{O}$ bond length is $2.7 \AA$, which results in a porous structure of $1.67 \mathrm{~nm}$ periodicity. ${ }^{9} \mathrm{On}$ $\mathrm{Au}(111)$, the structure periodicity is $1.62 \pm 0.05 \mathrm{~nm}$, with the molecules in a dimer being spaced about $1 \mathrm{~nm}$ apart and an $\mathrm{OH} \cdots \mathrm{O}$ bond length of about $2.6 \AA$, similar to that in the solid phase. Here, the distortion of the unit cell is induced by the variations in the nearest-neighbor $\mathrm{Au}-\mathrm{Au}$ distances that exist in the reconstructed surface layer $\left(2.80 \AA\right.$ to $\left.2.87 \AA^{26}\right)$. On $\mathrm{Ag}(111)$ and $\mathrm{Cu}(100)$ surfaces, similar hexagonal arrangements are found with slightly larger periodicities (1.78 nm for TMA/
$\mathrm{Ag}(111),{ }^{27} 2.04$ and $2.28 \mathrm{~nm}$ for TMA $\left./ \mathrm{Cu}(100)^{28}\right)$ and therefore longer $\mathrm{OH} \cdots \mathrm{O}$ bond lengths. An increase in the $\mathrm{H}$ bond lengths on these surfaces is expected to yield weaker $\mathrm{H}$ bonds. Therefore, the reconstructed $\mathrm{Au}(111)$ surface provides a good match for adsorbed TMA molecules to form 2D hexagonal porous phases resembling the one found in the gas and solid phases. This may explain why $\mathrm{Au}(111)$ is the only substrate so far on which long-range assemblies of different packing densities of TMA, including the so-called "superflower" structure, were observed. $^{12}$

Spectroscopy of the TMA Nanoporous Network. To determine the electronic properties of the TMA porous network on $\mathrm{Au}(111)$, we rely on scanning tunneling spectroscopy (STS). In STS, the differential conductance $\mathrm{d} I / \mathrm{d} V$ is 


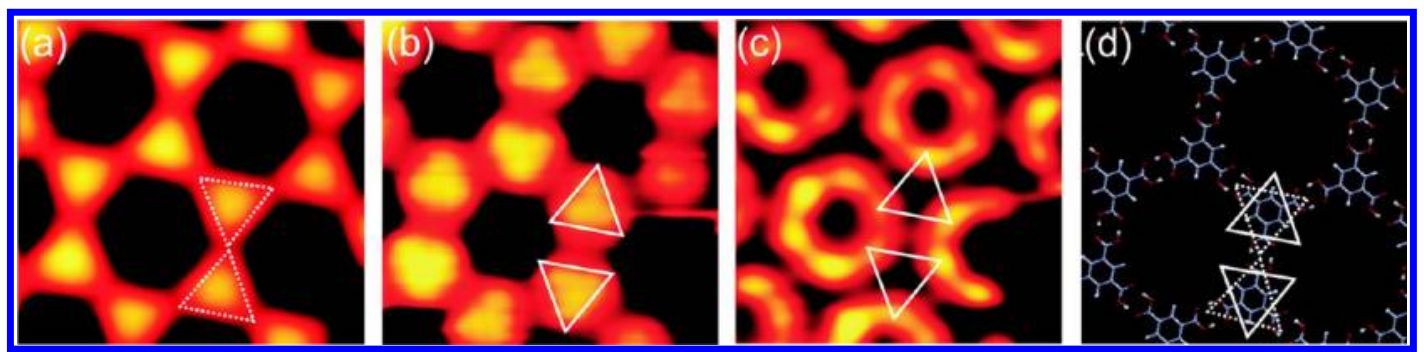

Figure 4. Orbital-mediated tunneling of TMA molecules. (a, b) STM images acquired at 1.0 and $2.7 \mathrm{~V}$, respectively. The shape of the molecule is highlighted by the dotted and solid triangles. (c) $\mathrm{dI} / \mathrm{dV}$ map recorded at $2.7 \mathrm{~V}$. The solid triangles are pasted from panel b. (d) Chemical drawing of the TMA cyclic dimer structure with the two triangular appearances superimposed.

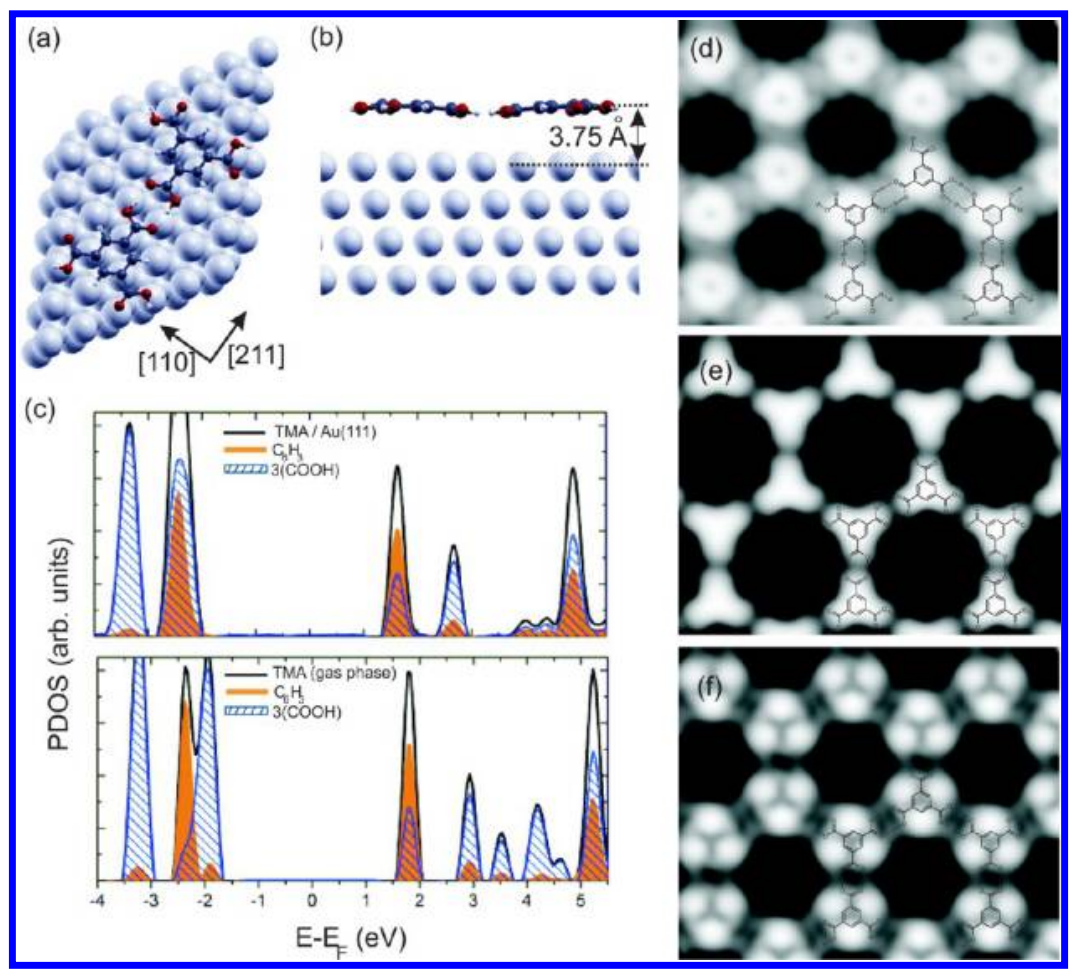

Figure 5. (a) Top-view illustration of the unit cell used in the calculations containing a TMA $R_{2}^{2}(8)$ dimer. (b) Side-view illustration of the fully relaxed TMA dimer on the $\mathrm{Au}(111)$ slab. (c) Projected density of states (PDOS) of the TMA molecule in the gas phase and adsorbed on Au(111) as shown in image a; PDOS of the aromatic core $\left(\mathrm{C}_{6} \mathrm{H}_{3}\right)$ and the three COOH groups is indicated. (d, f) Simulated STM images for HOMO $(-2.5 \mathrm{~V})$ and LUMO $(1.5 \mathrm{~V})$. (e) Simulated STM image at $0.7 \mathrm{~V}$ inside the HOMO-LUMO gap. All STM images are simulated at a tip height of $3.5 \AA$.

proportional to the local density of states (LDOS) of the sample, probing filled (empty) states for negative (positive) applied bias voltage. Figure 3a illustrates the $\mathrm{d} I / \mathrm{d} V(V)$ spectra measured on the $\mathrm{Au}(111)$ surface, on the TMA molecules, and inside the pores. The sharp rise at $-0.5 \mathrm{~V}$ in the $\mathrm{Au}(111) \mathrm{d} I /$ $\mathrm{d} V(V)$ spectrum represents the onset of the surface state (SS) reminiscent of the $2 \mathrm{D}$ electron gas that resides on the $\mathrm{Au}(111)$ surface. $^{23}$ A similar feature can be observed in the TMA spectrum, which is shifted upward only a few tens of millivolts. The almost unperturbed SS detected on the molecule indicates that the molecules are physisorbed on the substrate and interact only weakly with it.

To highlight the differences in the spectra with respect to the bare $\mathrm{Au}(111)$ surface, we plot in the inset of Figure 3a the dI/ $\mathrm{d} V$ difference between the curve of the gold hcp region and the other curves. For completeness, we also include the $\mathrm{d} I / \mathrm{d} V$ difference between the hcp and fcc regions. It is expected that on bare $\mathrm{Au}(111)$ the hcp regions have an increased LDOS near the SS onset compared to the fcc regions as a result of the different degree of electron localization imposed by the reconstruction in the two regions. ${ }^{23}$ Although on the molecule the SS LDOS is lower than on the surface, in the pores we observe an energy redistribution of the LDOS: more electronic states are localized at energies around the Fermi level than at energies above $0.2 \mathrm{~V}$. This may be attributed to a partial electronic confinement due to a modified surface potential induced by the presence of the TMA superlattice. ${ }^{23}$ Stronger confinement of the SS electrons inside the pores may be realized on more reactive surfaces such as $\mathrm{Ag}$ and $\mathrm{Cu}^{7,8}$

The increase in the LDOS above $1.5 \mathrm{~V}$ measured at the pore is present on the TMA molecule as well and can be linked to unoccupied states of the molecule that are highly delocalized. To probe higher-energy states, we performed distance-voltage spectroscopy, which allows control over the tunneling current passing through the molecule and avoids irreversible changes to the molecules. ${ }^{14}$ Figure $3 \mathrm{~b}$ displays $\mathrm{d} z / \mathrm{d} V(V)$ spectra measured on the $\mathrm{Au}(111)$ surface and at two locations on the TMA molecule (center and side). The peak at $2.6 \mathrm{~V}$ detected only at 
the TMA locations is interpreted as the lowest unoccupied molecular orbital (LUMO) of the adsorbed TMA molecule. Note that the peak is more pronounced on the side of the molecule than at its center.

A direct visualization of the spatial extent of electronic charge densities of the molecule is possible when performing voltagedependent imaging. Figures 4a,b displays two topographies acquired at 1.0 and $2.7 \mathrm{~V}$, respectively. In both topographies, the molecules have a triangular shape that is highlighted by the dotted and solid triangles. In the first image, the molecule's triangular shape reflects the location of the $\mathrm{COOH}$ groups (based on the cyclic dimer organization), and in the second image, it is rotated by $60^{\circ}$. Now the triangle's corners are in line with the hydrogens of the benzene ring (Figure $4 \mathrm{~b}$ ). A structural drawing of the molecular arrangement together with the two triangular shapes is presented in Figure $4 \mathrm{~d}$. We argue that the observed change in the apparent shape is related to the electronic structure of the molecule, in particular, to the electronic state appearing in Figure $3 \mathrm{~b}$. The spatial distribution of the LDOS corresponding to this state can be visualized on an LDOS map (i.e., a spatial mapping of the $\mathrm{d} I / \mathrm{d} V$ signal). Figure $4 \mathrm{c}$ illustrates the LDOS map measured at $2.7 \mathrm{~V}$ and simultaneously recorded with the topography from Figure $4 \mathrm{~b}$. On the LDOS map, each TMA molecule is imaged as three bright lobes aligned with the hydrogens of the aromatic ring but further extended into the empty space of the pores. Here, the pores appear smaller, and their electronic structure is dominated by the extended molecular wave functions. This appearance of the molecule is observed only at voltages corresponding to the LUMO state and is suggestive of LUMOmediated electron tunneling from tip to substrate. LUMOmediated tunneling may also explain the appearances of TMA molecules on the graphite surface. ${ }^{11,13}$ An unambiguous interpretation of the molecular appearances in the STM images requires comparison with theoretical calculations. ${ }^{29}$

DFT-Based Modeling of the TMA Nanoporous Structures on $\mathrm{Au}(111)$. Figure 5a illustrates a top view of the unit cell used to model the porous structure adsorbed on $\mathrm{Au}(111)$. The molecules were placed on the surface with their centers close to a hollow site, according to the single-molecule calculation results. Given the large number of atoms in the molecule, we assume that there are many competing adsorption sites with small energy differences. A side view of the adsorption geometry of a fully relaxed TMA dimer on the surface is displayed in Figure $5 \mathrm{~b}$. The molecules are adsorbed with their aromatic centers at a distance of $3.75 \AA$ from the slab. $^{30}$ The molecules are oriented parallel to the $\mathrm{Au}(111)$ surface with a small degree of distortion introduced by the tilt of the $\mathrm{COOH}$ groups toward the gold surface. ${ }^{31}$ The $\mathrm{COOH}$ groups are adsorbed closer to the surface by $0.1-0.3 \AA$, indicating a stronger interaction with the surface than the center of the molecule.

Charge distribution analysis using the Bader charge analysis scheme was carried out for both the free and the adsorbed TMA molecules. On the free molecule, a significant charge redistribution occurs between the atoms of the $\mathrm{COOH}$ groups. The oxygens gain $\sim 2 e$ each, and the carbons and the hydrogens become positively charged. The charge transfer upon adsorption results in minor changes to this picture. After adsorption, each molecule gains a net charge of $0.06 e$ from the $\mathrm{Au}(111)$ surface. $^{32}$ This is consistent with the minor shift detected experimentally in the SS onset at the molecule location (Figure 3a). This implies that the depopulation of the surface state under the molecule is very small and reflects the weak molecule-substrate interaction.

The molecule-substrate bonding character may be regarded as partially electrostatic, mediated by the partially charged $\mathrm{COOH}$ groups, whereas the $\pi$ electrons of the aromatic ring are interacting with the substrate through weaker van der Waals forces. ${ }^{19,31,33}$ We calculated the adsorption energy of a TMA molecule on $\mathrm{Au}(111)$ in two cases (i.e., for an isolated molecule and as part of the H-bonded network using the vdW-DF functional). The adsorption energy of the isolated TMA is $E_{\mathrm{d}}=$ $3.6 \mathrm{eV}$, and for the $\mathrm{H}$-bonded molecule it drops to $E_{\mathrm{d}}=3.3$ $\mathrm{eV} .{ }^{34}$ The decreased affinity of $0.3 \mathrm{eV}$ in the latter case can be attributed to weaker interaction between the $\mathrm{COOH}$ groups and the surface because the $\mathrm{COOH}$ groups are now engaged in planar $\mathrm{H}$ bonds. Similar results have been reported for benzoic acid by Rochefort et al. ${ }^{31}$ The stabilization energy of the $\mathrm{R}_{2}^{2}(8)$ dimer in the adsorbed nanoporous structure is $9.98 \mathrm{kcal} / \mathrm{mol}$ (per $\mathrm{COOH}$ group), in very good agreement with other studies ( $10.01 \mathrm{kcal} / \mathrm{mol}$ for the gas-phase $\mathrm{R}_{2}^{2}(8) \operatorname{dimer}^{3}$ ).

To confirm the origin of the change in the appearance of the molecule in the experimental STM images, we simulated the STM images of the adsorbed molecules for bias voltages corresponding to the highest occupied and lowest unoccupied molecular orbitals (HOMOs and LUMOs) and for one value of the bias voltage in the gap. The calculated LDOS of an adsorbed TMA as part of the nanoporous network is displayed in Figure 5c together with the LDOS of gas-phase TMA. For better comparison with the experiment, the LDOS is displayed separately for the aromatic center and the $\mathrm{COOH}$ groups. The frontier orbitals of the adsorbed molecule are situated at $-2.5 \mathrm{~V}$ (HOMO) and $1.5 \mathrm{~V}$ (LUMO). The formation of the $\mathrm{H}$ bonded network mostly affects the HOMO of the unbounded molecule, which is now delocalized across the entire molecule rather than localized at the $\mathrm{COOH}$ groups as in the case of gasphase TMA. Although the HOMO appears to be almost equally distributed over the $\mathrm{COOH}$ groups and the $\mathrm{C}_{6} \mathrm{H}_{3}$ center, the LUMO has more weight on the $\mathrm{C}_{6} \mathrm{H}_{3}$ center than on the $\mathrm{COOH}$ groups. Figure $5 \mathrm{~d}-\mathrm{f}$ displays the calculated STM images corresponding to HOMO and LUMO as well as a bias voltage inside the HOMO-LUMO gap $(0.7 \mathrm{eV})$. Note that the calculated STM images should be only qualitatively compared to the experimental ones because the PBE functional is known to give not as good agreement with experiment in terms of energy gaps and level alignments. ${ }^{35,36}$ It can be clearly seen in Figure $5 \mathrm{~d}-\mathrm{f}$ that the appearance of the molecules in the STM images depends considerably on the bias voltage, in correspondence with the experimental results. For applied voltages corresponding to the HOMO-LUMO gap (Figure 5e), the triangular shape of the molecule is defined by the carboxylic group locations and is similar to the experimental image in Figure 4a. The latter is in agreement with the most reported appearance of TMA molecules in STM images in the literature. ${ }^{4,12,27,28}$ For applied voltages corresponding to the LUMO, the areas that display the highest charge density are located between the $\mathrm{COOH}$ groups (i.e., at the $\mathrm{H}$ locations on the aromatic ring). For visual guidance, the molecular structure of the molecules in the network is added to the simulated STM images. There is excellent agreement between the simulated STM images (Figure 5e,f) and the experimental STM images (Figure $4 a, b)$. We can conclude that the change in the appearance of the molecule originates from the different orbitals that mediate the electron tunneling through the molecule. Our findings illustrate that knowledge of the 
electronic properties of adsorbed molecules and their interactions is crucial for the interpretation of molecular appearances in STM images.

\section{CONCLUSIONS}

Nanoporous networks of TMA molecules are formed by selfassembly upon deposition on a $\mathrm{Au}(111)$ surface and are studied using STM and STS at low temperature. Analyses of the TMA superstructures support the idea that intermolecular $\mathrm{H}$ bonds are decisive in the formation of such porous patterns but also highlight the role of the substrate potential landscape that defines the intermolecular bond lengths and pattern periodicity. The preferential orientations of the TMA networks on the $\mathrm{Au}(111)$ surface give rise to peculiar domain boundaries that force molecules into unexpected bonding patterns. The electronic properties of both the molecules and the pores are investigated using STS and voltage-dependent STM imaging. The TMA molecular network imposes a weak confinement on the surface electrons into the pores whereas it dominates the electronic structure of the pores at LUMO energies through highly extended molecular wave functions. The appearance of the molecules in the STM images is strongly dependent on the applied bias voltage. In particular, a remarkable change in their appearance is detected for LUMO-mediated electron tunneling. Our observations are verified and complemented by DFTbased calculations of the nanoporous structure adsorbed on the $\mathrm{Au}(111)$ surface. The calculations demonstrate that the adsorbed porous structure is energetically favored compared to isolated molecules. The simulated STM images of the network are in excellent agreement with the experimental results.

\section{AUTHOR INFORMATION}

\section{Corresponding Author}

*E-mail: violeta.iancu@fys.kuleuven.be; Chris. VanHaesendonck@fys.kuleuven.be.

\section{Notes}

The authors declare no competing financial interest.

\section{ACKNOWLEDGMENTS}

We thank O. Ivasenko and S. De Feyter for fruitful discussions. This work has been supported by the Research Foundation Flanders (FWO, Belgium). For the computations, we used the infrastructure of the VSC - Flemish Supercomputer Center, funded by the Hercules Foundation and the Flemish Government, Department EWI.

\section{REFERENCES}

(1) Decker, R.; Schlickum, U.; Klappenberger, F.; Zoppellaro, G.; Klyatskaya, S.; Ruben, M.; Barth, J. V.; Brune, H. Using metal-organic templates to steer the growth of $\mathrm{Fe}$ and Co nanoclusters. Appl. Phys. Lett. 2008, 93, 243102.

(2) Elemans, J. A. A. W.; Lei, S.; De Feyter, S. Molecular and supramolecular networks on surfaces: from two-dimensional crystal engineering to reactivity. Angew. Chem. Int. Ed. 2009, 48, 7298-7333.

(3) MacLeod, J. M.; Ivasenko, O.; Fu, C.; Taerum, T.; Rosei, F.; Perepichka, D. F. Supramolecular ordering in oligothiophene-fullerene monolayers. I. Am. Chem. Soc. 2009, 131, 16844-16850.

(4) Lackinger, M.; Heckl, W. M. Carboxylic acids: versatile building blocks and mediators for two-dimensional supramolecular selfassembly. Langmuir 2009, 25, 11307-11321.

(5) Eder, G.; Kloft, S.; Martsinovich, N.; Mahata, K.; Schmittel, M.; Heckl, W. M.; Lackinger, M. Incorporation dynamics of molecular guests into two-dimensional supramolecular host networks at the liquid-solid interface. Langmuir 2011, 27, 13563-13571.

(6) Liu, J.; Chen, T.; Deng, X.; Wang, D.; Pei, J.; Wan, L.-J. Chiral hierarchical molecular nanostructures on two-dimensional surface by controllable trinary self-assembly. I. Am. Chem. Soc. 2011, 133, 2101021015.

(7) Lobo-Checa, J.; Matena, M.; Müller, K.; Dil, J. H.; Meier, F.; Gade, L. H.; Jung, T. A.; Stöhr, M. Band formation from coupled quantum dots formed by a nanoporous network on a copper surface. Science 2009, 325, 300-303.

(8) Klappenberger, F.; Kuhne, D.; Krenner, W.; Silanes, I.; Arnau, A.; de Abajo, F. J. G.; Klyatskaya, S.; Ruben, M.; Barth, J. V. Dichotomous array of chiral quantum corrals by a self-assembled nanoporous kagome network. Nano Lett. 2009, 9, 3509-3514.

(9) Kolotuchin, S. V.; Thiessen, P. A.; Fenlon, E. E.; Wilson, S. R.; Loweth, C. J.; Zimmerman, S. C. Self-assembly of 1,3,5-benzenetricalboxylic (trimesic) acid and its analogues. Chem.-Eur. J. 1999, 5, 2537-2547.

(10) Chui, S. S.-Y.; Lo, S. M.-F.; Charmant, J. P. H.; Orpen, A. G.; Williams, I. D. A chemically functionalizable nanoporous material $\left[\mathrm{Cu}_{3}(\mathrm{TMA})_{2}\left(\mathrm{H}_{2} \mathrm{O}\right)_{3}\right]_{n}$. Science 1999, 283, 1148-1150.

(11) Griessl, S.; Lackinger, M.; Edelwirth, M.; Hietschold, M.; Heckl, W. M. Self-assembled two-dimensional molecular host-guest architectures from trimesic acid. Single Mol. 2002, 3, 25-31.

(12) Ye, Y.; Sun, W.; Wang, Y.; Shao, X.; Xu, X.; Cheng, F.; Li, J.; Wu, K. A unified model: self-assembly of trimesic acid on gold. I. Phvs. Chem. C 2007, 111, 10138-10141.

(13) Nath, K. G.; Ivasenko, O.; MacLeod, J. M.; Miwa, J. A.; Wuest, J. D.; Nanci, A.; Perepichka, D. F.; Rosei, F. Crystal engineering in two dimensions: an approach to molecular nanopatterning. I. Phys. Chem. C 2007, 111, 16996-17007.

(14) Feng, M.; Zhao, J.; Petek, H. Atomlike, hollow-core-bound molecular orbitals of C60. Science 2008, 320, 359-362.

(15) Horcas, I.; Fernandez, R.; Gómez-Rodrguez, J. M.; Colchero, J.; Gómez-Herrero, J.; Baro, A. M. A software for scanning probe microscopy and a tool for nanotechnology. Rev. Sci. Instrum. 2005, 78, 013705.

(16) Braun, K.-F. Calculation of standing wave pattern and image processing program: STWS. http://www.kai-felix-braun.de/program. htm.

(17) Giannozzi, P.; Baroni, S.; Bonini, N.; Calandra, M.; Car, R.; Cavazzoni, C.; Ceresoli, D.; Chiarotti, G. L.; Cococcioni, M.; Dabo, I.; Dal Corso, A.; de Gironcoli, S.; Fabris, S.; Fratesi, G.; Gebauer, R.; Gerstmann, U.; Gougoussis, C.; Kokalj, A.; Lazzeri, M.; Martin-Samos, L.; Marzari, N.; Mauri, F.; Mazzarello, R.; Paolini, S.; Pasquarello, A.; Paulatto, L.; Sbraccia, C.; Scandolo, S.; Sclauzero, G.; Seitsonen, A. P.; Smogunov, A.; Umari, P.; Wentzcovitch, R. M. QUANTUM ESPRESSO: a modular and open-source software project for quantum simulations of materials. I. Phys.: Condens. Matter 2009, 21, 395502.

(18) Roman-Perez, G.; Soler, J. M. Efficient implementation of a van der Waals density functional: application to double-wall carbon nanotubes. Phvs. Rev. Lett. 2009, 103, 096102.

(19) Langreth, D. C.; Lundqvist, B. I.; Chakarova-Käck, S. D.; Cooper, V. R.; Dion, M.; Hyldgaard, P.; Kelkkanen, A.; Kleis, J.; Kong, L.; Li, S.; Moses, P. G.; Murray, E.; Puzder, A.; Rydberg, H.; Schröder, E.; Thonhauser, T. A density functional for sparse matter. I. Phys.: Condens. Matter 2009, 21, 084203.

(20) Perdew, J. P.; Burke, K.; Ernzerhof, M. Generalized gradient approximation made simple. Phvs. Rev. Lett. 1996, 77, 3865-3868.

(21) Tersoff, J.; Hamann, D. R. Theory of the scanning tunneling microscope. Phvs. Rev. B 1985, 31, 805-813.

(22) Kokalj, A. Computer graphics and graphical user interfaces as tools in simulations of matter at the atomic scale. Comput. Mater. Sci. 2003, 28, 155-168.

(23) Chen, W.; Madhavan, V.; Jamneala, T.; Crommie, M. F. Scanning tunneling microscopy observation of an electronic superlattice at the surface of clean gold. Phvs. Rev. Lett. 1998, 80, 14691472. 
(24) Repain, V.; Berroir, J. M.; Rousset, S.; Lecoeur, J. Interaction between steps and reconstruction on $\mathrm{Au}(111)$. Europhys. Lett. 1999, 47, 435-441.

(25) Ibach, H. Physics of Surfaces and Interfaces; Spinger: New York, 2006.

(26) Wang, Y.; Hush, N. S.; Reimers, J. R. Simulation of the $\mathrm{Au}(111)-(22 \times \sqrt{3})$ surface reconstruction. Phys. Rev. B 2007, 75, 233416.

(27) Payer, D.; Comisso, A.; Dmitriev, A.; Strunskus, T.; Lin, N.; Woll, C.; DeVita, A.; Barth, J. V.; Kern, K. Ionic hydrogen bonds controlling two-dimensional supramolecular systems at a metal surface. Chem.-Eur. J. 2007, 13, 3900-3906.

(28) Dmitriev, A.; Lin, N.; Weckesser, J.; Barth, J. V.; Kern, K. Supramolecular assemblies of trimesic acid on a $\mathrm{Cu}(100)$ surface. $I$. Phvs. Chem. B 2002, 106, 6907-6912.

(29) Sautet, P.; Bocquet, M.-L. Shape of molecular adsorbates in STM images: a theoretical study of benzene on $\mathrm{Pt}(111)$. Phys. Rev. B 1996, 53, 4910-4925.

(30) This binding distance is very close to $3.73 \AA$ as determined for a single TMA molecule adsorbed on a $5 \times 5$ unit cell; the singlemolecule bonding distance is $8.6 \%$ less than in the case when the PBE functional is used, which indicates that the inclusion of dispersive forces is important when treating weakly interacting systems such as TMA molecules adsorbed on low-reactivity Au surfaces. ${ }^{33}$

(31) Rochefort, A.; Wuest, J. D. Interaction of substituted aromatic compounds with graphene. Langmuir 2009, 25, 210-215.

(32) In the case of a single adsorbed molecule, the charge transfer is determined to be $0.12 e$.

(33) Abad, E.; Dappe, Y. J.; Martinez, J. I.; Flores, F.; Ortega, J. $\mathrm{C}_{6} \mathrm{H}_{6} / \mathrm{Au}(111)$ : interface dipoles, band alignment, charging energy, and van der Waals interaction. I. Chem. Phvs. 2011, 134, 044701.

(34) The vdW-DF functional typically yields the best agreement with the experiment but at overestimated interatomic distances. ${ }^{37}$ The use of true interatomic distances with the vdW-DF functional results in stronger van der Waals forces and hence larger binding energies. The adsorption energies are calculated using vdW-DF-optimized TMA coordinates and fixed gold atoms at a $2.88 \AA$ nearest-neighbor distances.

(35) The bias voltages used do not match exactly but reflect the energy regions where the experimental appearance corresponds to the theoretical appearance.

(36) Braun, K.-F.; Hla, S. W. Charge transfer in the TCNQsexithiophene complex. I. Chem. Phvs. 2008, 129, 064707.

(37) Li, G.; Tamblyn, I.; Cooper, V. R.; Gao, H.-J.; Neaton, J. B. Molecular adsorption on metal surfaces with van der Waals density functionals. Phys. Rev. B 2012, 85, 121409. 